\title{
Analyzing the spread of rule-breaking behavior, focusing on talking in class, based on decision matrices in a critical mass model with local interaction
}

\author{
Takuhiko DEGUCHI \\ Faculty of Education, Nara University of Education
}

\begin{abstract}
A questionnaire and a computer simulation were used to investigate the validity of a critical mass model of rulebreaking behavior with local interaction. In this model, individuals were only able to perceive some of their neighbors' behavior. The questionnaire assessed attitudes toward and the frequency of rule-breaking behavior, and 887 valid responses were obtained from Japanese junior high school students. Computer simulations based on cellular automata were conducted using the questionnaire data. The outputs of the simulations including local interactions showed strong positive correlations with the rule-breaking frequencies obtained with the questionnaire. These findings imply that models taking the limits of perception into account could be useful for describing real micro-macro relationships.
\end{abstract}

Key Words: critical mass model, decision matrix, social influence

\section{Introduction}

Many studies indicate that individuals might engage in rulebreaking depending on others' behaviors (e.g., Cialdini, Reno, \& Kallgren, 1990; Deguchi, 2018). Schelling (2006) proposes a critical mass model that enables analysis of the effects of others' behavior from both a micro and a macro perspective. Each individual has a "critical number," and will break a rule if the number of others doing so in his/her environment is equal to or exceeds his/her critical number. Thus, the total number of rule-breakers increases. This model predicts that rule breaking should spread in the manner of toppling dominoes. The critical mass model allows each individual to perceive all others' behaviors, and each always behaves according to his/her own critical number.

However, individuals cannot always perceive the behaviors of all others in their environment (Simon, 1961). To analyze local interactions between individuals' behaviors, computer simulations can be conducted, applying a cellular automaton model (e.g., Latané, Nowak, \& Liu, 1994; Takagi, 2006). In Takagi's (2006) study, each cell takes 1 of 2 states (e.g., "active" vs "inactive") and has its own critical rate (e.g., the minimum rate of surrounding rule breaking that will induce a conforming cell to break the rule). Cells change their state only according to their neighboring cells' states. The results show that a model limited to local interactions tends to produce fewer state changes.

In addition, Deguchi (2018) indicates that among students with deviant attitudes (i.e., with a critical number of 0 ), the mean score for rule breaking (of a possible range from 1 to $5 ; 1=\mathrm{I}$ did not do this at all to $5=\mathrm{I}$ did this frequently) is 2.90. This implies that people sometimes obey a rule in the real world even if they have a deviant attitude. The effects of this randomness in local interactions have been investi-

\footnotetext{
* Corresponding Author e-mail: deguchi@nara-edu.ac.jp Online Appendix: https://doi.org/10.2130/jjesp.1808
} 
gated with a simulation using a decision matrix (Deguchi, 2014) resembling a payoff matrix, as in game theory (e.g., Axelrod, 1998) and interdependence theory (e.g., Kelly et al., 2003). The values of M11, M12, M21, and M22 in the decision matrix (see Table 1) represent various individuals' attitudes toward 4 situations; each cell changes its state according to its own decision matrix. A cell sometimes (randomly) conforms to the behavior of the majority in its neighborhood without considering its own decision matrix. This randomness represents human ambiguity (Deguchi, 2014): for example, a cell is able to obey or break a rule regardless of whether its own attitude is deviant (M11<M21 and M12<M22) or obedient (M11>M21 and M12 $>$ M22). The results indicate that this type of randomness could increase the spread of rule-breaking behavior.

However, these studies do not provide information on the relationships between the outputs of simulations and the spread of real rule-breaking behavior, and it is not clear how well these models including local interaction or randomness can describe real phenomena. Therefore, in the present study, simulation outputs were compared with empirical questionnaire data to investigate the validity of these models.

Various kinds of rule-breaking behavior have been studied, such as littering (Cialdini et al., 1990) and talking in class (Deguchi \& Yoshida, 2005). In particular, talking out of turn is a common behavior in educational settings (Deguchi, 2018). Therefore, this study focused on students' talking in class about topics unrelated to the lesson.

\section{Method}

\section{Questionnaire}

There were 8 items in the questionnaire assessing talking in class. Four items (Deguchi, 2018) were used to measure attitudes for a decision matrix. Each student was required to choose the number best representing his/her degree of satisfaction with each of the 4 situations represented in the decision matrix, such as "you are whispering, but your neighbors are not" (M21). Responses were given on a seven-point scale ranging from 1 ("very unsatisfied") to 7 ("very satisfied").

The other 4 items of the scale assessing talking in class (e.g., "I joked or told funny stories irrelevant to the class") from the talking in class scale (Deguchi \& Yoshida, 2005) were used to measure the frequency of talking in class over the last month. The students answered on a five-point scale ranging from 1 ("I did not do this at all") to 5 ("I did this frequently").

Procedure. The questionnaire was administered twice at the same Japanese junior high school in order to collect data from many classrooms while controlling for some factors that could affect rule-breaking behavior (e.g., school size and location; Stewart, 2003), first in December 2012 (time 1), and second between October and November 2015 (time 2; these data were also analyzed in Deguchi, 2018). The questionnaire was distributed to 12 classes (4 per grade) at each time point. The number of students per class was approximately 40 . The total number of valid responses was 887 (boys: 433, girls: 447, unknown: 7; time 1: 448, time 2: 439).

The questionnaire was anonymous. Before answering, students were informed that their answers would not affect their grades and that they could skip questions they did not want to answer. Institutional approval was obtained before data collection.

\section{Calculating Rule-breaking Rates}

Simulations were conducted using a set of rules based on Deguchi's (2014) model applied to a decision matrix (Table 1) that differed for each cell (Figure A, see Online Appendix). The method of the simulations was nearly the same as in Deguchi (2014) except for the following; a) Rules 3-1 and 3-2 were modified, b) the questionnaire data were used as input (M11-M22), and c) both the local and global interactions were investigated. Rules 2, 3-1, and 3-2 enabled each cell to change its state on the basis of either its neighbors' behaviors (Rule 3-1) or its own attitudes (Rule 3-2) using the "scores" calculated in Rule 1 . The probability of using Rule 3-2 is referred to as "M-prob." M-prob represents human ambiguity (Deguchi, 2014). In the present study, the value of M-prob was set at .25, .50, .75, or 1.00 , because there is a J-curved relationship between M-prob and rulebreaking rate (Deguchi, 2014). The values of M11, M12, M21, and M22 in the decision matrix represent individual differences, and cells differed from each other in these values. Therefore, to avoid too much complexity in the model, M-prob was fixed at the same value for every cell in each simulation executed, as in Deguchi (2014).

For the local (interaction) condition, 441 cells were arranged in a $21 \times 21$ matrix to use Moore neighborhood ( 8 directly adjacent cells) as in Deguchi (2014). For the global condition, cells were arranged in a one-dimensional matrix 
Deguchi: Analyzing the spread of rule-breaking behavior based on decision matrices in a critical mass model with local interaction

Table 1 Rules of simulation and decision matrix (adapted from Deguchi (2014), p. 441, Tables 1 and 2)

Rule 1

Impact of $\mathrm{O}$ on a cell=the number of obeying cells in the neighborhood.

Impact of $\mathrm{B}$ on a cell=the number of rule-breaking cells in the Neighborhood.

Score of $\mathrm{O}$ on a cell $=\mathrm{M} 11 \times$ impact of $\mathrm{O}+\mathrm{M} 12 \times$ impact of $\mathrm{B}$

Score of $\mathrm{B}$ on a cell $=\mathrm{M} 21 \times$ impact of $\mathrm{O}+\mathrm{M} 22 \times$ impact of $\mathrm{B}$

Rule 2

The cells change their states in accordance with Rule 3-1 or Rule 3-2 per step. The probability of using Rule 3-2 is called "M-prob." The M-probs of all the cells are the same. The rule used is determined for each cell.

Rule 3-1

If impact of $\mathrm{O}$ is higher than impact of $\mathrm{B}$, then the cell changes to obeying.

If impact of $\mathrm{B}$ is equal to or higher than impact of $\mathrm{O}$, then the cell changes to rule breaking.

Rule 3-2

If score of $\mathrm{O}$ is higher than score of $\mathrm{B}$, then the cell changes to obeying.

If score of $\mathrm{B}$ is equal to or higher than score of $\mathrm{O}$, then the cell changes to rule breaking.

\begin{tabular}{rrcc}
\hline Decision matrix & You/Neighbor(s) & Obeying & Breaking \\
& Obeying & M11 & M12 \\
& Breaking & M21 & M22 \\
\hline
\end{tabular}

Note. Rules 1 and 2 are the same as used in Deguchi (2014), while Rules 3-1 and 3-2 differ. The rules of the present study mean that a cell changes its state to rule-breaking when impact/score B is equal to impact/score O. However, in Deguchi (2014), the cell does not change in this case.

containing the same number of cells as the number of decision matrices collected from the corresponding class. The entire matrix was considered to represent each cell's neighborhood (that is, each cell could perceive all the other cells' states). When M-prob=1.00, this model is equivalent to the critical mass model. In neither condition was the matrix a torus, because this would not accurately represent a classroom.

Simulations were conducted separately for each class. Each cell assumed either an "obeying" or a "rule-breaking” state at any given time point. At the first time step, the states of all the cells were identical; that is, the initial rule-breaking rate was set at 0 or $100 \%$. Subsequently, 199 further steps were simulated; at each step, every cell either changed or maintained its state. The final rule-breaking rate (the percentage of rule-breaking cells in the matrix at the 200th step) was taken as the output index of the simulation.

For each simulation, decision matrices were allocated randomly to locations in the class from the relevant pool of data sets, which were composed of the questionnaire data (seven-point ratings for the 4 situations M11, M12, M21, and M22). First, the available pool was reordered randomly. The required number of matrices to fill each cell was then drawn from the pool in order, returning to the start of the list when necessary (this was always required for a $21 \times 21$ matrix). Finally, all cells in the matrix were assigned to random locations. This almost exactly followed the process used by Takagi (2004).

The above process, from allocation of decision matrices to calculation of the final rule-breaking rate, was executed 100 times per condition. Therefore, the total number of simulations in each condition was 2,400 (100 simulations $\times 24$ classes). The simulation was written in Microsoft Visual Basic .NET and was a modified version of the program used by Deguchi (2014).

\section{Results and Discussion}

Item scores for frequency of talking in class were summed (Cronbach's $\alpha=.88$ ) and the sum was divided by 4 (the number of items) to produce a score ranging from 1 to 5 for each participant (mean $=2.54, S D=0.93$ ). Each cell's critical rate was calculated using the relevant formula (Table 2). Figure 1 indicates final rule-breaking rates (global condition, M-prob=1.00) and the relationship between current and estimated new rule-breaking rates reckoned from the 
Table 2 Formulae for calculating critical rate

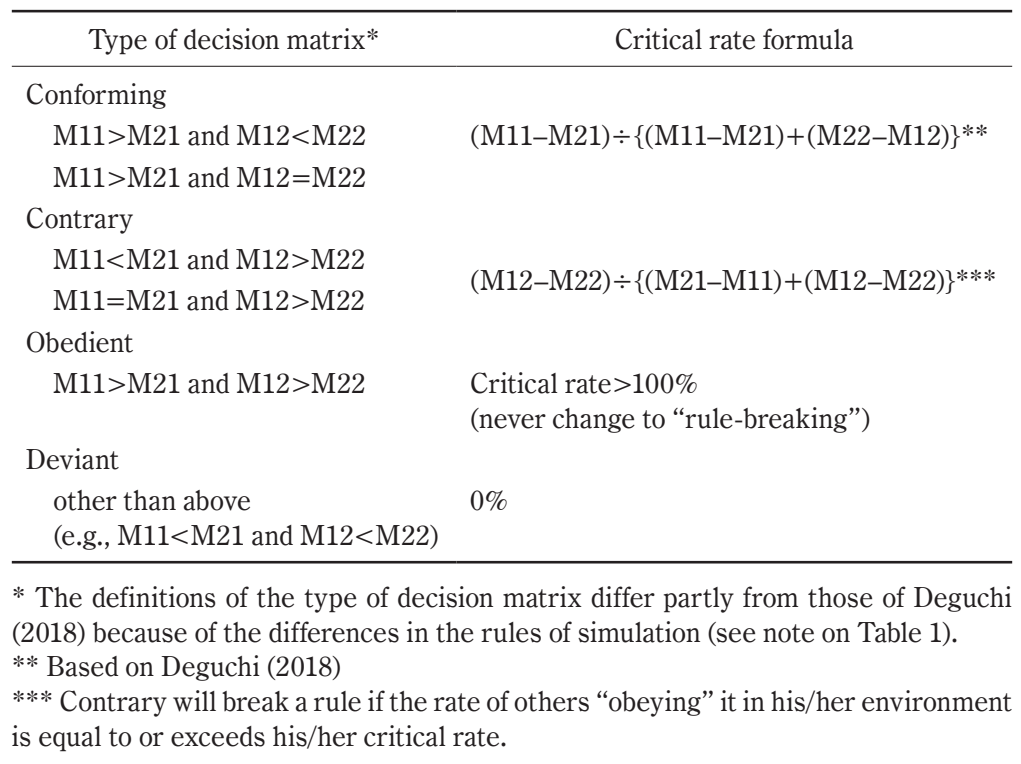

Grade 1
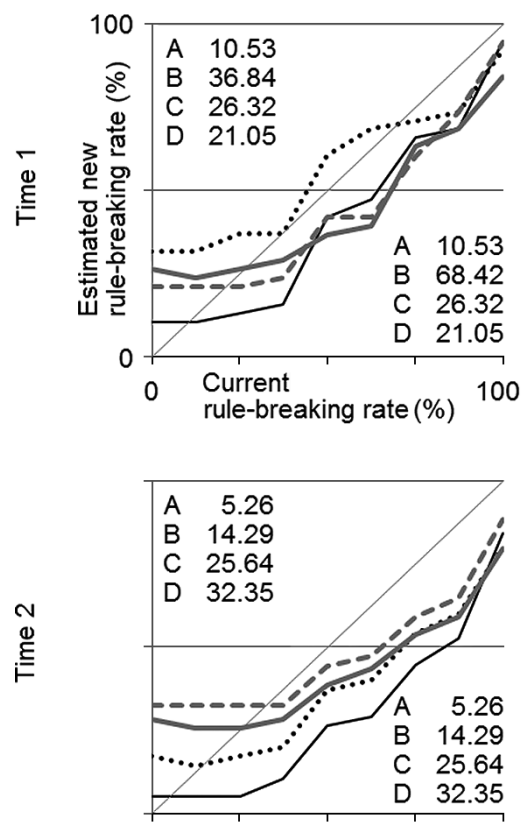

Grade 2
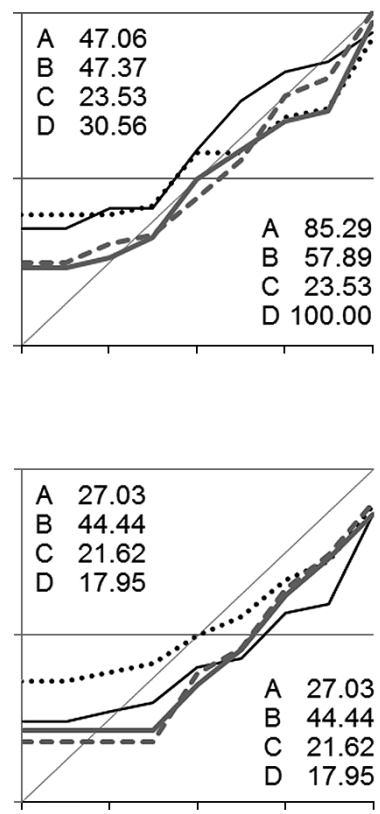

Grade 3
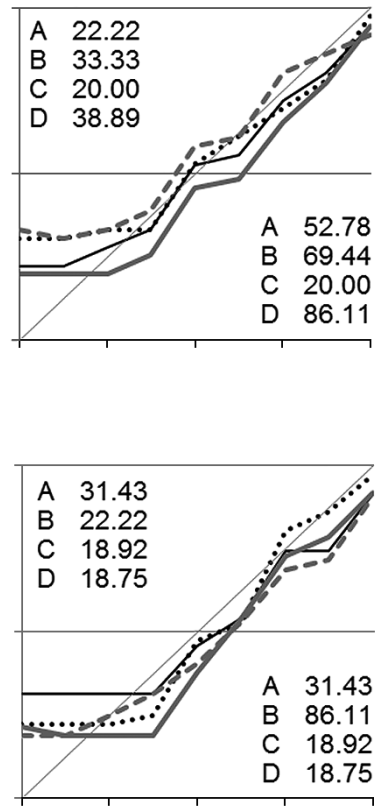

Figure 1 The relationship between critical rate and rule-breaking rate for each class.

Note. A-D each represent a single class in no particular order. Values at the upper left of each panel represent final rule-breaking rates when initial rule-breaking $=0 \%$; values at the lower right represent those when initial rule-breaking rates $=100 \%$ (global condition, M-prob=1.00). 
Deguchi: Analyzing the spread of rule-breaking behavior based on decision matrices in a critical mass model with local interaction

critical rates. Some classes' curves intersected twice with the 45-degree line with points that represents approximate convergence. In these classes, the final rule-breaking rate was lower when the initial rule-breaking rate was $0 \%$ than when it was $100 \%$.

Table 3 shows the correlations between the mean rulebreaking frequency as measured with the questionnaire ( $n=32-39)$ and final rule-breaking rate in the simulation $(n=100)$ for each class. ${ }^{1)}$ Many significant positive correlations were identified; these tended to be stronger in the local condition than in the global condition. In particular, Pearson correlation coefficients with an initial rule-breaking rate of $100 \%$ were greater than .65 ( $p \mathrm{~s}<.01)$. When $\mathrm{M}$-prob $=1.00$, the correlation was significantly stronger $(p=.00)$ in the local $(r=.71)$ than the global condition $(r=.45)$.

In addition, for the global condition with an initial rulebreaking rate of 100 , the correlation $(r=.66)$ was stronger $(p=.04)$ when $\mathrm{M}$-prob=.75 than when $\mathrm{M}-\mathrm{prob}=1.00$ $(r=.45)$. In contrast, for the local condition with an initial rule-breaking rate of 0 , the correlation $(r=.44)$ was weaker $(p=.02, p=.03)$ at $\mathrm{M}-\mathrm{prob}=.25$ than at M-prob $=1.00(r=.61)$ or $.75(r=.60)$.

These results imply that a model incorporating local interaction or ambiguity (i.e., $0<\mathrm{M}$-prob $<1$ ) might describe real micro-macro relationships; that is, the model could have validity although the cells perceive the statuses (obeying/rule-breaking) of fewer others or sometimes do not consider their own attitudes (their decision matrices). However, the validity of the model with local interaction decreases when M-prob is low. This decrease might be caused by dilution of the effects of the decision matrices on the simulation outputs.

\section{Acknowledgements}

I would like to express my gratitude to the students and teachers for their cooperation with the questionnaire and

Table 3 Correlations between simulation outputs and questionnaire data

\begin{tabular}{|c|c|c|c|c|c|c|c|c|}
\hline Type of interaction & \multicolumn{8}{|c|}{ Local } \\
\hline M-prob & \multicolumn{2}{|c|}{.25} & \multicolumn{2}{|c|}{.50} & \multicolumn{2}{|c|}{.75} & \multicolumn{2}{|c|}{1.00} \\
\hline Initial rule-breaking rate $(\%)$ & 0 & 100 & 0 & 100 & 0 & 100 & 0 & 100 \\
\hline$r$ & $.44^{*}$ & $.65^{* *}$ & $.57 * *$ & $.72 * *$ & $.60^{* * *}$ & $.68^{* *}$ & $.61^{* *}$ & $.71^{* *}$ \\
\hline$r_{s}$ & $.55^{* *}$ & $.68^{* *}$ & $.57 * *$ & $.69 * *$ & $.57 * *$ & $.70^{* *}$ & $.52 * *$ & $.68^{* *}$ \\
\hline mean & 35.95 & 96.47 & 48.47 & 71.77 & 42.50 & 53.59 & 34.88 & 55.62 \\
\hline$S D$ & 38.18 & 2.35 & 34.14 & 26.84 & 25.55 & 27.16 & 16.83 & 23.01 \\
\hline Type of interaction & \multicolumn{8}{|c|}{ Global } \\
\hline M-prob & \multicolumn{2}{|c|}{.25} & \multicolumn{2}{|c|}{.50} & \multicolumn{2}{|c|}{.75} & \multicolumn{2}{|c|}{1.00} \\
\hline Initial rule-breaking rate (\%) & 0 & 100 & 0 & 100 & 0 & 100 & 0 & 100 \\
\hline$r$ & $.51^{*}$ & $.65^{* *}$ & $.49^{*}$ & $.59 * *$ & $.48^{*}$ & $.66^{* *}$ & $.45^{*}$ & $.45^{*}$ \\
\hline$r_{s}$ & $.41^{*}$ & $.73^{* *}$ & .34 & $.63^{* *}$ & .35 & $.68 * *$ & .38 & $.49 *$ \\
\hline mean & 6.14 & 93.74 & 12.32 & 82.60 & 18.99 & 48.45 & 26.57 & 40.22 \\
\hline$S D$ & 1.99 & 2.89 & 4.29 & 17.78 & 6.81 & 34.12 & 10.94 & 28.18 \\
\hline
\end{tabular}

$* p<.05, * * p<.01$

Note 1. $N=24$. Means are the averages of the mean rule-breaking rate calculated per class, and $S D$ s represent degrees of differences among the 24 classes.

Note 2. Spearman's rank correlation coefficients were also calculated in order to investigate whether outlier data were present. However, there were no large differences between rank $\left(r_{s}\right)$ and product-moment $(r)$ correlation coefficients, and signs of outliers were not found.

1) The result of additional analysis conducted separately for data collected at each time can be seen Table A in Online Appendix. 
to Takeshi Imura, Naoyuki Taniguchi, and my colleagues for supporting the research. I would also like to thank Editage (www.editage.jp) for English language editing. This research was supported by JSPS KAKENHI Grant Numbers JP22730508, JP26380885.

\section{References}

Axelrod, R. (1998). The Evolution of Cooperation. (H. Matsuda, Trans.) Kyoto: Minerva shobou. (Original work published 1984)

Cialdini, R. B., Reno, R. R., \& Kallgren, C. A. (1990). A focus theory of normative conduct: Recycling the concept of norms to reduce littering in public places. Journal of Personality and Social Psychology, 58, 10151026. doi: 10.1037/0022-3514.58.6.1015

Deguchi, T. (2014). A simulation of rule-breaking behavior in public places. Social Science Computer Review, 32, 439-452. doi: 10.1177/0894439313511186

Deguchi, T. (2018). Normative consciousness, principles, and frequency of talking in class: Focusing on withinand between-level factors. Japanese Journal of Experimental Psychology, 57, 93-104. doi: 10.2130/jjesp.1705 (In Japanese)

Deguchi, T., \& Yoshida, T. (2005). The relationships among normative consciousness, individual traits, and frequency of whispering during college lectures: Focusing on adjustment to college life. Japanese Journal of Social
Psychology, 21, 160-169. doi: 10.14966/jssp.KJ00003978688 (In Japanese)

Kelly, H. H., Holmes, J. H., Kerr, N. L., Reis, H. T., Rusbupt, C. E., \& Van Lange, P. A. M. (2003). An atlas of Interpersonal Situations. Cambridge, England: Cambridge University Press.

Latané, B., Nowak, A., \& Liu, J. H. (1994). Measuring emergent social phenomena: Dynamism, polarization, and clustering as order parameters of social systems. Behavioral Science, 39, 1-24. doi: 10.1002/bs.3830390102

Schelling, T. C. (2006). Micromotives and Macrobehavior (with a New Preface and the Nobel Lecture). New York: W.W. Norton \& Co.

Simon, H. A. (1961). Administrative Behavior: A Study of Decision-making Processes in Administrative Organization (Second Edition). New York: The Macmillan Company.

Stewart, E. A. (2003). School social bonds, school climate, and school misbehavior: A multilevel analysis. Justice Quarterly, 20, 575-604.doi: 10.1080/07418820300095621

Takagi, E. (2004). Critical Mass Model, Continued. Retrieved from http://eiji-takagi.la.coocan.jp/class/prog/041203c mass.html (February 18, 2017) (In Japanese, translated by the author of this article)

Takagi, E. (2006). A computational model of social 'critical mass' phenomena. Saitama University Review (Faculty of Liberal Arts), 42, 55-62. (In Japanese) 